XLV. Jahres-Versammlung der Schweizerischen Gesellschaft für Dermatologie und Venereologie am 28. und 29. September 1963 in Basel

Dermatologica, 1964;129:57

\title{
Zur Histogenetik der hereditären Epidermolysen
}

\begin{tabular}{|l|l|}
\hline U.W. & Schnyder \\
\hline T. & Šalamon \\
\hline
\end{tabular}

Aus der Dermatologischen Klinik der Universität Zürich (Direktor: Prof. Dr. med. $H$. Storck)

Adresse der Autoren: Dr. U. W. Schnyder und Dr. T. Šalamon, Dermatologische Klinik der Universität, Zürich (Schweiz) Anhand von 38 Exzisionen aus gesunder und blasiger Haut von Patienten mit Epidermolysis bullosa simplex Koebner, Epidermolysis bullosa dystrophica s. hyperplastica Cockayne-Touraine und Epidermolysis bullosa dystrophica polydysplastica Hallopeau-Siemens nehmen die Autoren zur Pathogenese der Blasenbildung Stellung. Beim Koebnerschen Typ ist sie intraepithelial, beim Hallopeau-Siemensschen Typ streng subepidermal. In Abhängigkeit vom Schweregrad findet man bei letzterem auch in der klinisch gesunden Haut eine Epithelatrophie sowie fakultativ eine verminderte Anfärb-barkeit der Elastica im Papillarkörper. Die CockayneTourainesche Form hingegen nimmt histopathologisch eine Mittelstellung ein.

Die Arbeit wird später ausführlicb. publiziert.

5 Dermatologica

Vol. 129

No. 1 (1964) 